\title{
Optical generation and detection of elastic waves in solids
}

\author{
D. Royer, M.-H. Noroy and M. Fink
}

Laboratoire Ondes et Acoustique, Université Paris VII, URA 1503 du CNRS, ESPCI, 10 rue Vauquelin, 75231 Paris cedex 05, France

\begin{abstract}
In this paper we review optical methods suitable to generate and to detect elastic waves in solids without any mechanical contact. The mechanisms underlying elastic wave generation by the impact of a pulsed laser beam are outlined. The principles of optical techniques able to detect surface displacements of amplitude less than one angstrom are described. Examples of applications in domains such as nondestructive testing and acoustic field imaging are given.
\end{abstract}

\section{1- INTRODUCTION}

Since they do not require any mechanical contact, laser techniques offer an attractive alternative to conventional piezoelectric transducers for generating and probing elastic waves. The objective of this paper is to review the principles underlying both optical generation and detection processes. Applications in domains such as nondestructive testing of materials and acoustic field imaging will be described.

The combination of laser generation with optical detection provides a completely remote ultrasonic system for material inspection. In the field of nondestructive testing, the thermoelastic regime is needed to avoid any surface damage and the low induced amplitude makes optical detection difficult. Recently, techniques that use an array of thermoelastic sources have been proposed. Results obtained in our laboratory with a 16-beam YAG laser are given.

The optical detection is much less sensitive than the piezoelectric transducer, but it presents many advantages: the vibrating surface can be investigated at a distance without disturbing the acoustic field, the measurement is local and can be calibrated from optical wavelength, the bandpass is wide. Furthermore, as there is no mechanical contact, a large area can be scanned by moving either the object or the light beam. Examples of local measurements of transient surface displacements of guided elastic waves, generated by piezoelectric and photothermal techniques will be presented. A high resolution optical system developed in our laboratory for imaging pulsed ultrasonic fields transmitted in water by piezoelectric transducer is described.

\section{2- LASER IMPACT GENERATION OF ELASTIC WAVES}

Elastic wave generation in solids by pulsed laser irradiation was first suggested by R.M. White [1]. The advent of high power pulsed lasers has allowed the photothermal generation to be implemented. Theoretical models of the generation mechanisms have been developed [2-5]. Compared to ultrasonic techniques using piezoelectric transducers, the photothermal generation presents many avantages: no mechanical contact is needed, the position and the shape of the source can be modified, elastic waves can be generated in solids at elevated temperature [6]. Fields of application of this technique are: nondestructive testing [7-8], material evaluation [9], acoustic emission and microscopy. Most of the experiments has been performed by irradiating a solid with laser pulses. Elastic waves are detected either by conventional (piezoelectric, capacitive, electro-magneto-acoustic) transducers or by optical methods. 
According to the optical power density deposited on a free surface, laser generation of elastic waves proceeds by mechanisms that may be classified in two main categories: those involving a modification of the irradiated surface (ablation regime) and those that do not (thermoelastic expansion). Assuming optical pulses of low power density, such that the solid is not caused to melt, the source due to thermal expansion is characterized by two force dipoles parallel to the free surface (Fig. 1a). At higher power density, such that melting and vaporization occur, momentum transfer results in the removal of material from the solid. This ablation regime is characterized by forces normal to the irradiated surface (Fig. $1 \mathrm{~b}$ ). The emission characteristics of laser-generated-elastic waves can be improved by using an array of thermoelastic sources.

\section{2-1- Thermoelastic regime.}

When a low power laser pulse strikes onto a solid surface, one part of the incident energy is reflected, the other part is absorbed and heat converted. The fast temperature rise, localized near the surface produces a thermal expansion which in turn creates a transient elastic stress field.

Temperature distribution. In a metal such as duraluminum, skin depth $(\approx \mathrm{nm})$ is much smaller than thermal diffusion length $(\approx \mu \mathrm{m})$ and acoustic wavelength $(\approx \mathrm{mm})$. The depth of the thermoelastic source is governed by the diffusion process. The temperature distribution can be obtained by solving the heat diffusion equation [10]. Figure 2 shows the temperature rise, at the center, in a duraluminum sample irradiated by a laser pulse of profile $\mathrm{q}(\mathrm{t})=\left(\mathrm{t} / \tau^{2}\right) \mathrm{e}^{-\mathrm{t} / \tau}$ (duration $\Delta=2,5 \tau=25 \mathrm{~ns}$, absorbed energy $\mathrm{Q}=$ $1 \mathrm{~mJ}$ ) having a gaussian spatial distribution (diameter $1 \mathrm{~mm}$ ). At any time, no significant temperature rise exists at distance from the surface more than $10 \mu \mathrm{m}$. During the laser pulse the diffusion depth reaches $2 \mu \mathrm{m}$, which is much smaller than the laser beam width and the acoustic wavelength. The thermoelastic source is confined in a thin disk within the surface. The local heating vanishes $400 \mathrm{~ns}$ after the laser impact. Pulses arriving at a rate less than $1 \mathrm{MHz}$ can be treated as independent.

Bulk wave generation. The transient temperature rise generates a dilatation $\Delta V=(3 \alpha / \rho C) Q H(t)$ of the heated volume $\mathrm{V}$, proportional to the absorbed heat $\mathrm{Q}$ ( $\alpha$ is the linear thermal expansion coefficient) and having a step dependence $\mathrm{H}(\mathrm{t})$. This dilatation creates stresses which can be expressed, in an isotropic solid (Lamé constants $\lambda$ and $\mu$ ), as $\Delta \mathrm{T}_{\mathrm{ij}}=(3 \lambda+2 \mu)(\alpha / \rho \mathrm{C})(\mathrm{Q} / \mathrm{V}) \mathrm{H}(\mathrm{t}) \delta_{\mathrm{ij}}$. The displacement created by this source localized in the volume $V$ is given by a time-convolution [11]:

$$
u_{n}\left(x_{i}, t\right)=\int_{V} \Delta T_{i j}\left(\xi_{i}, t\right) * G_{n i, j}\left(x_{i}, \xi_{i}, t\right) d V\left(\xi_{i}\right)
$$

where $G_{n i}\left(x_{i}, \xi_{i}, t\right)$ is the Green function giving the $n$-th displacement component at the observation point $x_{i}$ at time $t$, due to an impulse force parallel to the $x_{i}$-axis applied at point source $\xi_{i}$ at time $t=0$. This relation simplifies if the source is assumed to be a point located at the origin:

$$
u_{n}\left(x_{i}, t\right)=M_{i j}(t) * G_{n i j}\left(x_{i}, 0, t\right) \quad \text { where } \quad M_{i j}(t)=\int_{V} \Delta T_{i j}\left(\xi_{i}, t\right) d V\left(\xi_{i}\right)
$$

is the sismic momentum describing the strength of the point source. In an isotropic solid this tensor is reduced to a scalar $M(t)=(3 \lambda+2 \mu)(\alpha / \rho C) Q H(t)$. Setting $g_{n}=G_{n i, i}$, the displacement components are given by

$$
\left.\mathrm{u}_{\mathrm{n}}\left(\mathrm{x}_{\mathrm{i}}, \mathrm{t}\right)=\Gamma \mathrm{H}(\mathrm{t}) * \mathrm{~g}_{\mathrm{n}}\left(\mathrm{x}_{\mathrm{i}}, 0, \mathrm{t}\right)=\Gamma \mathrm{g}_{\mathrm{n}} \mathrm{H}_{\mathrm{i}}, 0, \mathrm{t}\right) \text { with } \Gamma=(3 \lambda+2 \mu)(\alpha / \rho \mathrm{C}) \mathrm{Q}
$$

$\mathrm{g}_{\mathrm{n}}{ }^{\mathrm{H}}\left(\mathrm{x}_{\mathrm{i}}, 0, \mathrm{t}\right)$ is the Green function corresponding to a step-like excitation. The displacement amplitude is proportional to the absorbed energy i.e, in the thermoelastic regime to the incident optical energy.

A thermoelastic source localized at the free surface of a solid creates only tangential forces. So a point source is equivalent to a set of two orthogonal and horizontal force dipoles modeling the surface center of expansion (SCOE) of the solid due to the laser heating [5].

At a distance $R$, mechanical displacements launched by this source can be classified in two types: displacements detected around wave-front arrival times $\mathrm{aR}$ and $\mathrm{bR}$, respectively of longitudinal and shear 
waves (velocities $c_{L}=a^{-1}$ and $c_{S}=b^{-1}$ ) and those observed between $a R$ and $b R$ and after $b R$. These two parts are respectively far field and near field contributions of the laser generated displacements. In the far field, longitudinal and shear waves are uncoupled: the displacement vanishes between $a R$ and $b R$. In the near field, longitudinal and shear waves are coupled: a continous variation between the two arrival times aR et $\mathrm{bR}$ is observed.

In the near field, along $\mathrm{z}$-axis, i.e. at the epicentre with respect to the source, the displacement is normal to the free surface. Figure 3 referred to a material such as duraluminum $\left(k=c_{L} / c_{S}=2\right)$. First, a step-like depression is observed at time $\mathrm{t}=\mathrm{aR}$ corresponding to the solid retraction along the normal, followed by $\mathrm{a}$ low frequency motion and by a step-like elevation at time bR. After this wavefront, the sample tends to an equilibrium state different from the initial state since the step-like source still expands. The slow variation between times $\mathrm{aR}$ and $\mathrm{bR}$ characterizes the coupling between $\mathrm{L}$ and $\mathrm{S}$ waves in the near field.

Wave-front expansion. Directivity patterns. Rose has calculated the Green function of a point source when the observation times are closed to the arrival times of the wave-fronts [5]:

- the radial displacement decreases as $1 / R: g_{R}{ }^{H}(R, \theta, t)=\Lambda a R^{-1} A(\theta) \delta(t-a R)+O\left(R^{-2}\right)$ with $\Lambda=\left(\pi \rho c_{L}{ }^{2}\right)^{-1}$.

The directivity function $A(\theta)$ is real for any angle $\theta$ with respect to the normal to the surface and the time dependence of the longitudinal wave is the same as that of the laser pulse

$$
A(\theta)=\frac{\sin \theta \sin 2 \theta\left(k^{2}-\sin ^{2} \theta\right)^{1 / 2}}{\left(k^{2}-2 \sin ^{2} \theta\right)^{2}+2 \sin \theta \sin 2 \theta\left(k^{2}-\sin ^{2} \theta\right)^{1 / 2}}, \quad k=\frac{c_{L}}{c_{S}}
$$

- for the transverse displacement: $g_{\theta}^{H}(R, \theta, t)=\frac{1}{2} \Lambda b R^{-1}\left[B_{1}(\theta) \delta(t-b R)-\frac{B_{2}(\theta)}{\pi(t-b R)}\right]+O\left(R^{-3 / 2}\right)$, the directivity function

$$
B(\theta)=B_{1}(\theta)+i B_{2}(\theta)=\frac{\sin 2 \theta \cos 2 \theta}{\cos ^{2} 2 \theta+2 \sin \theta \sin 2 \theta\left(k^{-2}-\sin ^{2} \theta\right)^{1 / 2}}
$$

is purely real for $\theta<\theta_{c}=\sin ^{-1}\left(c_{S} / c_{L}\right)$ and complex for $\theta>\theta_{c}$. Because of the head wave creation the near field term varies as $R^{-3 / 2}$ when $\theta>\theta_{c}$ and as $R^{-2}$ when $\theta<\theta_{c}$ as for the longitudinal displacement.

Given the additional term $[\pi(t-b R)]^{-1}$, the time dependence of the shear wave differs from that of the laser pulse.

The computed point-source directivity patterns are plotted in Fig. 4. They are symmetric with respect to the normal to the surface and no emission exists along this direction. Longitudinal waves are radiated in a single lobe with a maximum in direction closed to $65^{\circ}$ in the case of duraluminum. For shear waves, the displacement is maximum at $\theta=30^{\circ}$ and vanishes at $45^{\circ}$, then the polarities of the two lobes are opposited.

Rayleigh waves. Since the source is localized within the surface, the thermoelastic expansion generated Rayleigh waves with a great efficiency. Figure 5 shows the surface waveform detected by a capacitive transducer. The Rayleigh pulse is bipolar with a time duration that is proportional to the transit time of the acoustic wave accross the source, i.e. to the width of the laser beam. Cielo et al [12] used an axicon lens to produce an annular thermoelastic source in order to increase the amplitude at the focus. The mechanical displacement was measured by a Michelson interferometer.

\subsection{Surface modification and treatment.}

The efficiency of the generation can be improved by increasing the optical power or by coating the solid surface with an absorbing film or with a transparent plate.

Material ablation. At increased optical power densities $\mathrm{W}_{0}$ the solid surface is caused to melt and to evaporate. Momentum transfer produced by removal material gives rise to a force normal to the surface. For duraluminum and a $20-\mathrm{ns}$ laser pulse duration, ablation occurs at Wo $>15 \mathrm{MW} / \mathrm{cm}^{2}$. From the presence of a liquid or gazeous phase at the solid surface, the absorption coefficient varies (up to $90 \%$ ) 
versus the incident power. Experimentally, Ready [10] has shown that most of the material was removed in a liquid phase. This effect greatly increases the normal force. At the end of the laser pulse, ablation will continue over a long time, until the surface temperature goes down to the ablation threshold.

The directivity patterns of the point normal force source can be computed by the method of Miller and Pursey [13]. Longitudinal waves are mostly radiated at the epicentre (Fig. 6 a) and the ablative source is omni-directionnal but non-isotropic. For shear waves (Fig. 6 b), the source, in the case of duraluminum, is more efficient at angles closed to $35^{\circ}$.

In practice, the optical power density may be raised by focusing the light beam. Figure 7 shows typical signals detected at the epicentre by capacitance transducer for increasing power densities. The first waveform corresponds to an incident power density just above the ablation threshold: the thermoelastic contribution is preponderant. For increasing power densities, the amplitude of the longitudinal arrival increases whereas the step at shear wave arrival time vanishes progressively. It should be noticed that the longitudinal displacement undergoes a maximum and hereafter decreases. This phenomenon can be ascribed to plasma shiclding which prevents part of the optical energy from reaching the surface. The fast rise after the ablation threshold comes from the increase in the absorption coefficient due to the surface melting.

The amplitude of Rayleigh waves generated in the ablation regime is also increased. The pulse shape, compared to the signal in Fig. 5, is symmetric with respect to a mirror normal to the axis passing through the middle of the pulse. The difference is explained by the fact that in thermoelastic regime, the surface moves towards the outside and in the ablation regime, it moves towards the inside.

Absorbing film. The energy absorption can be increased by coating the surface with various liquid layer (oil for example): the film temperature rise causes it to evaporate. The induced momentum transfer creates a normal force as in the ablation regime. This effect favours longitudinal wave generation [14]. Rayleigh waves may also be enhanced by the evaporation of a liquid coating, without any propagation damping if the film is deposited only on the irradiated area of the surface.

Constraining layer. A glass slide rigidly bonded to the irradiated surface changes the boundary conditions $[1,15]$. Assuming that the absorption takes place at the interface with the substrate, the expansion source is now buried. The acoustic waveform depends on the thickness of the plate with respect to the time duration of the laser pulse.

\section{2-3- Generation by an array of thermoelastic sources}

Recently, techniques that use an array of photothermal sources or continous excitation by a moving source have been proposed to improve the directivity of the thermoelastic generation and to increase the amplitude of the laser-generated elastic waves [16]. In our laboratory, a 16-beam YAG laser has been used to implement a phased array of thermoelastic sources on the solid surface [17]. The emission time of each laser pulse is properly delayed to achieve at a chosen point in the sample (the focus) a constructive summation of each acoustic pulse. The acoustic field at the focus is obtained by adding the waveforms generated by each line source with the proper time delays $\tau_{i}=t_{0}-r_{i} / c_{L} ; r_{i}$ is the distance between the center of an element and the focal point and $t_{0}$ the arrival time of all the delayed wave fronts. Figure 8 shows the directivity pattern of an array of 16-thermoelastic sources of aperture $20 \mathrm{~mm}$, focusing longitudinal waves into the point $\theta=64^{\circ}, R=50 \mathrm{~mm}$. The symmetry of diagramm in Fig. 4 a is broken and a narrow ultrasonic beam is obtained.

Figure 9 shows the result of experiments carried out with a multiple beam YAG-laser, manufactured by the french company B.M. Industries, providing 16 optical pulses of 30-ns duration. Each laser beam is focused into a line of $8-\mathrm{mm}$ height at a distance of $1 \mathrm{~m}$. The longitudinal wave displacement transmitted through a duraluminum half-cylindrical sample (24-mm radius) was detected by an optical probe. In the direction $\theta=65^{\circ}$ where the constructive summation occurs (Fig. 9 a), the peak value of the signal reaches $4 \mathrm{~nm}$. In the direction $\theta=-65^{\circ}$, each acoustic pulse arrives with such delays that they are time-resolved (Fig. 9 b). The ratio of amplitudes received in this two symmetrical directions is nearly 15 which is consistent with the number of sources and the cylindrical propagation factor. With this technique a significant improvement in the signal-to-noise ratio of a laser-based ultrasonic system can be achieved. Furthermore, for noncontact nondestructive applications, the direction of the ultrasonic beam can be electronically controlled by changing the time delay law [18]. 


\section{3- OPTICAL DETECTION}

The different techniques can be placed in two main categories. The first category refers to noninterferometric methods, mainly based on the deflexion of a narrow optical beam by the local variation of the slope of the surface or on the diffraction by phase surface grating of an optical beam which overlaps several acoustic wavelengths. The second category refers to interferometric methods based on the measurement of the phase or of the frequency modulation of the light by the normal component of the displacement.

\section{3-1. Noninterferometric probes}

The deflexion method, also called knife edge technique has been extensively used to visualize surface acoustic wave fields [19]. Its main interest is to permit the scanning of the surface. As shown in figure 10, the laser beam focused onto the surface is deflected by the acoustic wave ripple. The knife edge (in practice the edge of the photodiode) masking partially the oscillating beam, the photodiode output current is modulated. The amplitude of the angular deviation of thereflected beam is proportional to the local slope of the probed surface. This efficient and simple technique is exploited in two instruments: the AFM (Atomic Force Microscope) and the SLAM (Scanning Laser Acoustic Microscope [20]) used for non- destructive testing. In this application, a fluid insures the transmission of ultrasound to the mirror surface, the surface of which is scanned by the probe beam. The disadvantages of the knife edge technique are that it requires a good surface state and that the sensitivity decreases in high frequency domain. The highest frequency is obtained when $d=\lambda$, i.e. $20 \mathrm{MHz}$ for a focal length $F=25 \mathrm{~cm}$, a He-Ne laser $(\Lambda=633 \mathrm{~nm}, \varnothing=1 \mathrm{~mm})$ and a wave velocity $\mathrm{V}=3000 \mathrm{~m} / \mathrm{s}$.

Diffraction by surface grating. If the optical beam overlaps several acoustic wavelengths $(d \gg>\lambda)$, the surface corrugation behaves as a phase grating [21]. For a given angle of incidence $\theta_{0}$, the optical beam is diffracted into beams of frequencies $\Omega \pm \mathrm{m} \omega$, along directions such that : $\sin \theta_{\mathrm{m}}=\sin \theta_{0} \pm \mathrm{m} \Lambda / \lambda$. The power of the first order beam is proportional to the square of the mechanical displacement. Thus the measurement of the relative intensities of the diffracted beams of order 1 and 0 provides the spatial distribution of the Rayleigh wave power [22]. This technique is usable only when the diffracted beams are distinguishable, namely at high frequencies ( $\mathrm{f}>100 \mathrm{MHz}$ ) and in a steady regime. As the photocurrent is proportional to $\mathrm{u}^{2}$, the sensitivity for small displacements is low.

\subsection{Interferometric probes.}

These probes are able to measure any mechanical displacement normal to a surface in a continous or in a transient regime [23]. The phase $\Phi$ of a light beam (wave number $K=2 \pi / \Lambda$ ) back-reflected by the vibrating surface of an objet is modulated by the normal surface displacement $u \cos (\omega t+\varphi)$ :

$$
\Delta \Phi=2 \mathrm{Ku} \cos (\omega \mathrm{t}+\varphi) \quad, \quad \mathrm{K}=2 \pi / \Lambda
$$

As optical detectors are quadratic, for it to be exploited, this phase modulation has to be either directly converted into an amplitude modulation of the photocurrent with the aid of a homodyne interferometer (Michelson) or transposed into a phase modulation of the current in the RF domain by heterodyne interferometry, that means, with a change of the optical frequency. If the acoustic wave frequency is sufficiently high (a few $\mathrm{MHz}$ ), the information on the mechanical displacement, contained in the spectrum of the optical signal, can be extracted directly by optical spectroscopy with a Fabry-Pérot type interferometer used as a frequency discriminator [24].

Homodyne interferometer. The first method consists in mixing the probe beam $\mathrm{S}$ with a reference beam $\mathrm{R}$ coming from the same laser source (Fig. 11). The beams S and R are superposed and directed to the photodetector. The photocurrent produce by the beating of the two waves is given by

$$
\mathrm{I}=\mathrm{I}_{0}\left\{1+\cos \left[2 \mathrm{Ku} \cos (\omega \mathrm{t}+\varphi)+\Phi_{\mathrm{S}}-\Phi_{\mathrm{R}}\right]\right\}
$$

To reduce the effect of the random fluctuations of the phases $\Phi_{S}$ and $\Phi_{R}$, due to low frequency thermal and mechanical disturbances, the reference mirror is displaced so that the phase difference $\Phi_{S}-\Phi_{R}$ is maintained constant (stabilized Michelson interferometer). As in a standard Michelson interferometer, the laser power is divided, by a beam splitter, into two equal parts which reflect respectively on the object 
(probe beam S) and on the mirror (reference beam R) and then mix on the photodetector (Fig. 11). The intensity of the photocurrent resulting from the beating of beams $R$ and $S$ depends sinusoiddally on the optical path difference $L_{S}-L_{R}$ with a period $\Lambda$. Maximum sensitivity is obtained for $L_{S}-L_{R}=(n \pm 1 / 4) \Lambda$ $\rightarrow \Phi_{S}-\Phi_{R}= \pm \pi / 2$. In order to maintain the operating point in this phase quadrature position, the position of the reference mirror is controlled by a piezoelectric actuator driven by the low frequency part $(\mathrm{f}<1 \mathrm{kHz})$ of the detected signal $[25,26]$. The limited dynamic range of the piezoelectric control necessitates periodical adjustments. For this phase-quadrature operating point and small displacements $(\mathrm{Ku} \ll 1: \mathrm{Ku}=0.01$ for $\mathrm{u}=1 \mathrm{~nm}$ and $\mathrm{a} \mathrm{He}-\mathrm{Ne}$ laser) the photocurrent intensity is given by

$$
\mathrm{I}=\mathrm{I}_{0}[1+2 \mathrm{Ku} \cos (\omega \mathrm{t}+\varphi)]
$$

The sensitivity is limited by the shot noise from the d.c. part $\mathrm{I}_{0}$ from the photocurrent. For an electronic detection bandwidth $\mathrm{B}$, the mean square value of the noise current intensity is given by $2 \mathrm{eI}_{0} \mathrm{~B}$ where $\mathrm{e}$ is the electron charge. The minimum measurable displacement $\mathrm{u}_{\text {min }}$, corresponding to a signal-tonoise ratio equal to 1 , is found to be

$$
\mathrm{u}_{\min }=\frac{\Lambda}{2 \pi} \sqrt{\frac{\mathrm{eB}}{\mathrm{I}_{0}}} \quad \Rightarrow \quad \mathrm{u}_{\min }=2.3 \times 10^{-5} \AA / \sqrt{\mathrm{Hz}}
$$

for a 2-mW He-Ne laser $\left(I_{0}=0.3 \mathrm{~mA}\right)$ and $\mathrm{B}=1 \mathrm{~Hz}$.

Heterodyne probes. The feature in this technique is the introduction of a frequency shifter, usually an acousto-optic Bragg-cell, in either (or both) arm(s) of the interferometer. Let $f_{B}$ be the acoustic wave frequency (typically $40-100 \mathrm{MHz}$ ) in the Bragg-cell. In the expression (7) of the photocurrent, a term at the frequency $\mathrm{f}_{0}$ equal to $\mathrm{f}_{\mathrm{B}}$ or $2 \mathrm{f}_{\mathrm{B}}$ appears in the alternative part

$$
\left.i(t)=I_{0} \cos \left[\omega_{0} t+2 K u \cos (\omega t+\varphi)+\Phi_{S}-\Phi_{R}\right]\right\}
$$

The optical phase modulation of the probe beam by the mechanical displacement is transposed into the RF domain. The spectrum of $i(t)$ comprises a central line at $f_{0}$ and lateral lines at $f_{0} \pm m f$ whose heights are given by Bessel functions $J_{m}(2 K u)$. If the displacement $u$ is small $(K u \ll 1)$, i.e. less than $200 \AA$, the spectrum reduces to the carrier at $f_{0}$ and two lateral lines at $f_{0} \pm f\left(F i g .12\right.$ a). The ratio $R=(K u)^{-1}$ of the level of the carrier and of one side component provides the absolute mechanical vibration in steady state regime independently of the light power reflected by the sample : $u(\AA) \approx 1000 / \mathrm{R}$ for a He-Ne laser.

Compact optical configuration. The devices constructed so far are modified Michelson interferometers in which the beam splitter is an acousto-optic Bragg-cell [19]. A Mach-Zehnder type of interferometer is preferable [27]. As shown in Fig. 12 b, the two beams are extracted by the beam splitter cube (BS) from the horizontally-polarized laser beam. The reference beam $R$ is directed through a prism towards the photodiode. The probe beam $S$ whose frequency is shifted by a colinear Bragg cell $\left(\mathrm{f}_{B}=70 \mathrm{MHz}\right)$ is reflected by the sample. After passing twice the quarter wavelength plate this beam is vertically polarized and reflected by the polarizing beam splitter PBS, along the direction of the reference beam. The two beams pass through the analyser and beat on the photodetector. The photocurrent at frequency $f_{B}$ is phase modulated by the vibration of the sample. The off-centering, with respect to the axis of the two cubes, eliminates the spurious signals coming from the reflexions of the $S$ and $R$ beams (points $a$ and $b$ ) on PBS cube. Since the optical part is compact (less than $40 \mathrm{~cm}$ long, laser included) the stability is improved [27].

Coherent electronic detection. The random phase fluctuations $\Phi_{S}-\Phi_{R}$ affect the carrier and the sidebands in the same way. Their effect can be cancelled or strongly reduced by coherent electronic detection [28]. Figure 13 shows a broad bandwidth processing suitable for transient regime [29] which provides a signal $\mathrm{s}(\mathrm{t})=\sin [2 \mathrm{Ku} \cos (\omega \mathrm{t}+\varphi)]$ proportional to the mechanical displacement $u \cos (\omega t+\varphi)$ if $\mathrm{Ku} \ll 1$.

Time delay or velocity interferometry. In the previously described homodyne or heterodyne interferometers, the wave reflected by the sample surface, which can have a distorted wave front, beats with a plane reference wave. Since these probes are not able to collect more than one speckle, their sensitivity is reduced on rough surfaces. A solution to this problem is to use only one optical wave issued from the 
surface. The signal is obtain by mixing this wave with itself after a time delay $\tau$. Since the distorted wavefronts of the two waves are matched together, this technique is efficient on diffusing surfaces. As shown in Fig. 14, a Michelson interferometer can be used to make the beatings. The delay can be insured by a multiple path device, an optical fiber [30] or a Fabry-Pérot interferometer [24]. Setting $4 \pi u(t) / \Lambda$ the phase-shift induced by the surface displacement, at the same time, the phase-shift undergoes by the time delayed optical wave is $4 \pi u(t-\tau) / \Lambda$. According to Eq. 7 , the photocurrent is given by

$$
\mathrm{I}=\mathrm{I}_{0}\{1+\cos [2 \mathrm{~K}[\mathrm{u}(\mathrm{t})-\mathrm{u}(\mathrm{t}-\tau)]+2 \pi v \tau+\phi]\}
$$

where $\phi$ is a phase-shift introduced in one of the arms in order to maintain the interferometer in the phase quadature condition: $2 \pi v \tau+\phi= \pm \pi / 2$. Assuming small displacements $(\mathrm{u}<<\Lambda)$, the a.c component is given by

$$
\mathrm{i}(\mathrm{t})=\mathrm{I}_{0} \sin \{2 \mathrm{~K}[\mathrm{u}(\mathrm{t})-\mathrm{u}(\mathrm{t}-\tau)]\} \cong 2 \mathrm{KI} \mathrm{I}_{0}[\mathrm{u}(\mathrm{t})-\mathrm{u}(\mathrm{t}-\tau)]
$$

This technique can operate in two different ways: i) the time-delay $\tau$ is larger than the signal duration $\Theta$, then $u(t-\tau)=0$ when $u(t) \neq 0$ and the photocurrent is proportional to the normal displacement $u(t) ;$ ii) $\tau<<$ $\Theta$, then $u(t)-u(t-\tau) \cong \tau d u / d t$ and the photocurrent is proportionnal to the normal velocity $v(t)$. The time delay interferometer operates as a Doppler velocimeter. Assuming an incident optical wave $E$ exp i $\Omega$ t on the moving surface, the angular frequency of the backscattered light field $E$ exp i[St $+4 \pi u(t) / \Lambda]$ expressed as $\Omega[1+2 v(t) / c]$. This Doppler shift gives rise to lateral components in the optical spectrum.

The interferometer developed by Monchalin [24] uses a confocal Fabry-Pérot of length $50 \mathrm{~cm}$ (Fig. 15 a). The bandwidth of the cavity was $1.5 \mathrm{MHz}$ or $10 \mathrm{MHz}$ according to the reflectivity of the mirrors. Its length was controlled with the aid of piezoelectric actuators so as to maintain the laser frequency on the slope of the transmission peak of the cavity which operates as a frequency discriminator (Fig. $15 \mathrm{~b}$ ).

\subsection{Experimental results}

We will present here a few results to illustrate the possibilities of the compact heterodyne interferometer developed in our laboratory and manufactured by B.M. Industries (optical probe SH 120).

Figure 16 deals with the optical excitation and detection of Lamb waves propagating in a duraluminum hollow cylinder (outer diameter $20 \mathrm{~mm}$, thickness $0.5 \mathrm{~mm}$ ). The modes of propagation are those in a plate: a symmetrical $\mathrm{S}_{0}$ (antisymmetrical $\mathrm{A}_{0}$ ) mode whose longitudinal (transverse) component is preponderant, for the modes without cut-off frequency. The velocity of the first mode is higher than that of the second. The YAG laser beam (pulse duration $80 \mathrm{~ns}$, energy $8 \mathrm{~mJ}$ ) is focused along a line (length $15 \mathrm{~mm}$, width 0.1 $\mathrm{mm}$ ). The probe beam, diametrically opposite to the source detects the normal component of the displacement. The first signal corresponds to the symmetrical mode with small normal displacement, the second to the flexural mode $\mathrm{A}_{0}$ whose normal displacement is very large. The strong dispersive effect in the propagation of this mode clearly appears: the wave train expands as it propagates. For large times, the slope of the instantaneous phase versus the time inverse provides the thickness of the tube [31]. The theoretical wave form, computed from the Rayleigh-Lamb equation is in good agreement with experimental data (Fig. 16 b).

Sismic prospecting experiments can be simulated either numerically by computing theoretical models or physically in the laboratory by using scaled down physical model. Concerning "physical modeling", the main problems are: i) the scaling down, with piezoelectric transducers, of the small size of the emitters and receivers with respect to the acoustic wavelength, ii) the coupling between the model and the transducers. Laser ultrasonic techniques provide nearly point source and point detector with no mechanical contact with the model. Figure 17 a shows the experimental set-up and the model made of lucite and duraluminum. The point source is located on one of the lucite surface, $2.5 \mathrm{~mm}$ from the interface. The detection point is moved on the opposite surface. Longitudinal $(P)$ and shear $(S)$ bulk waves, Rayleigh waves $(R)$ and head waves can be observed on the signals displayed in Fig. 17 b [32].

The ultrasonic pulse-echo technique is widely used in nondestructive testing and medical diagnosis. The improvement of the lateral resolution is generally achieved by focusing the acoustic beam and by increasing the frequency of the transducer. Practically, a beam width less than $0.5 \mathrm{~mm}$ requires a central frequency up 
to $15 \mathrm{MHz}$. Calibration methods using a miniature hydrophone or a reflective ball target are limited in the frequency domain to $10 \mathrm{MHz}$ and have a poor spatial resolution $(0.4 \mathrm{~mm})$. We have developed an optical imaging system having a broadband spatial and temporal resolution, which allows absolute measurements of the beam parameters without disturbing the acoustic field [33]. The experi-mental set-up (Fig. 18 a) includes a thin plastic membrane or pellicle (diameter $45-100 \mathrm{~mm}$, thickness $3-6 \mu \mathrm{m}$ ) immersed in a water tank in front of the transducer and the compact heterodyne interferometer previously described. To record the acoustic field, the transducer is moved in two directions parallel to the pellicle by stepping motors. Figure $18 \mathrm{~b}$ shows the 3-dimension profile of the transient acoustic field launched in water by a $25-\mathrm{MHz}$ focused transducer $(\mathrm{F}=18 \mathrm{~mm})$ probed on a $6-\mu \mathrm{m}$ thick membrane located in the focal plane. The spatial resolution is $20 \mu \mathrm{m}$ and the detection bandwidth reaches $40 \mathrm{MHz}$. Since the optical heterodyne process makes the measurements insensitive to environmental disturbances, such as low frequency motions of water, the minimum detectable displacement ( $1 \AA$ ) is as small as in the air.

\section{4- CONCLUSION}

The purpose of this paper was to review optical means of generation and detection of elastic waves in solids not requiring any mechanical contact. Concerning laser generation, the physical limitations of the single point source could be overcome by implementing an array of thermoelastic sources providing timedelayed acoustic pulses. Significant improvement in the beam paramaters has been obtained with a 16-beam YAG laser. Concerning optical detection, heterodyne probes are easy to calibrate and have a broad detection bandwidth and a low sensitivity to environmental noise. So they are suitable for acoustic field mapping. Velocimetry interferometry, using for example a confocal Fabry-Pérot, which is capable to collect many speckles should be preferred for measurements on rough surfaces.

\section{REFERENCES}

[1] - White R.M., J. Appl. Phys., 34 (1963) 3359.

[2] - Scruby C.B., Dewhurst R.J., Hutchins D.A. and Palmer S.B., J. Appl. Phys., 51 (1980) 6210.

[3] - Hutchins D.A., Dewhurst R.J. and Palmer S.B., J. Acoust. Soc. Am. 70 (1981) 1362

[4] - Hutchins D.A., "Ultrasonic Generation by Pulsed Laser", in Physical Acoustics, Vol.18, chap. 2. Edited by W.P. Mason and R.N. Thurston, Academic Press (1988).

[5] - Rose L.R.F., J. Acoust. Soc. Am., 75 (1984) 723.

[6] - Calder C.A., Draney E.C. and Wilcox W.W., J. Nuclear. Mat., 97 (1981) 126.

[7] - Scruby C.B. and L.E. Drain, "Laser Ultrasonics Techniques and Applications", Adam Hilger (1990)

[8] - Cooper J.A., Crosby R.A., Dewhurst R.J., Mc Kie A.D.W. and Palmer S.B., IEEE. Trans. UFFC33 (1986) 462.

[9] - Castagnede B., Kim K.Y., Sachse W. and Thompson M.O., J. Appl. Phys. , 0 (1991) 150

[10]-Ready J.F., "Effect of High Power Radiation"; Academic Press, New-York (1971).

[11]- Aki K. and Richards G., Quantitative Seismology, Freeman, San Francisco (1980) Vol. 1, Chap. 6.

[12]- Cielo P., Jen C.K. and Maldague X., Can. J. Phys., 64 (1986) 1324.

[13]- Miller G.F. and Pursey H., Proc. Roy. Soc. London, A 223 (1954) 521.

[14]- Dewhurst R.J., Hutchins D.A., Palmer S.B. and Scruby, C.B., J. Appl. Phys. 53 (1982) 4064.

[15]- Von Gutfeld R.J., Ultrasonics, (1980) 175.

[16]- Berthelot Y.H. and Busch-Vishniac I.J., J. Acoust. Soc. Am., 81 (1987) 317.

[17]- Noroy M.-H., Royer D. and Fink M., J. Acoust. Soc. Am., 94 (1993) 1934

[18]- Noroy M.-H., Royer D. and Fink M., IEEE Ultrasonics Symposium Proc., Baltimore (nov. 1993).

[19]- Whitman R.L. and Korpel A., Applied Optics, 8, (1969) 1567.

[20]- Kessler L.W. and Yuhas D.E., Proc. IEEE, 67, (1979) 526.

[21]- Stegeman G.I., IEEE Trans. Son. Ultrason. SU-23, (1976) 33.

[22]- Slobodnik A.J., Proc. IEEE, 58 (1970) 488.

[23]- Monchalin J.P., IEEE Trans. Ultrason. Ferroelectric and Freq. Control, UFFC-33, (1986) 485.

[24]- Monchalin J.P., Appl. Phys. Lett., 47, (1985) 14.

[25]- Kwaaitaal Th., Rev. Sci. Instrument, 45 (1974) 39.

[26]- Kroll M., and Djordjevic B.B., IEEE Ultrason. Symp. Proc., (1982) 864.

[27]- Royer D., Dieulesaint E. and Martin, Y. IEEE Ultrason. Symp. Proc. (1985) 432 
[28]- De La Rue R.M., Humphryes R.F., Mason I.M. and Ash e.A. Proc. IEE 119 (1972) 117.

[29]- Royer D., and Dieulesaint E., IEEE Ultrason. Symp. Proc., (1986) 527.

[30]- Bowers J.E., Jungerman R.L., Khuri-Yakub B.T. and Kino G.S.,J. Lightwave Tech.,1 (1983) 429

[31]- Royer D., Dieulesaint E. and Leclaire Ph., IEEE Ultrason. Symp. Proc. (1989) 1163.

[32]- Pouet B., "Modélisation physique par ultrasons laser. Application à la modélisation sismique". Thèse de 1'Université Paris 7, 10 avril 1991.

[33]- Royer D., Dubois N. and Fink M., Appl. Phys. Lett., 61, (1992) 153.

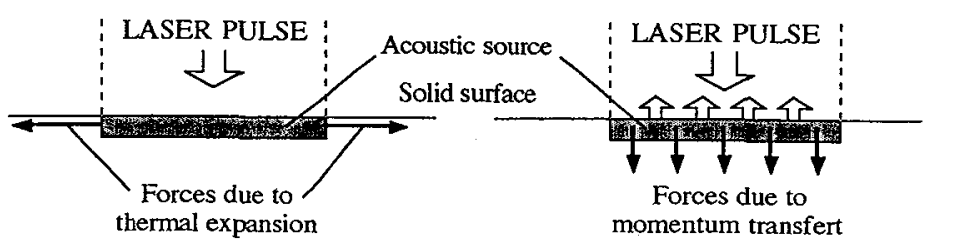

Fig. 1. Laser impact generation.

a) Thermoelastic regime.

b) Ablation regime.

a)
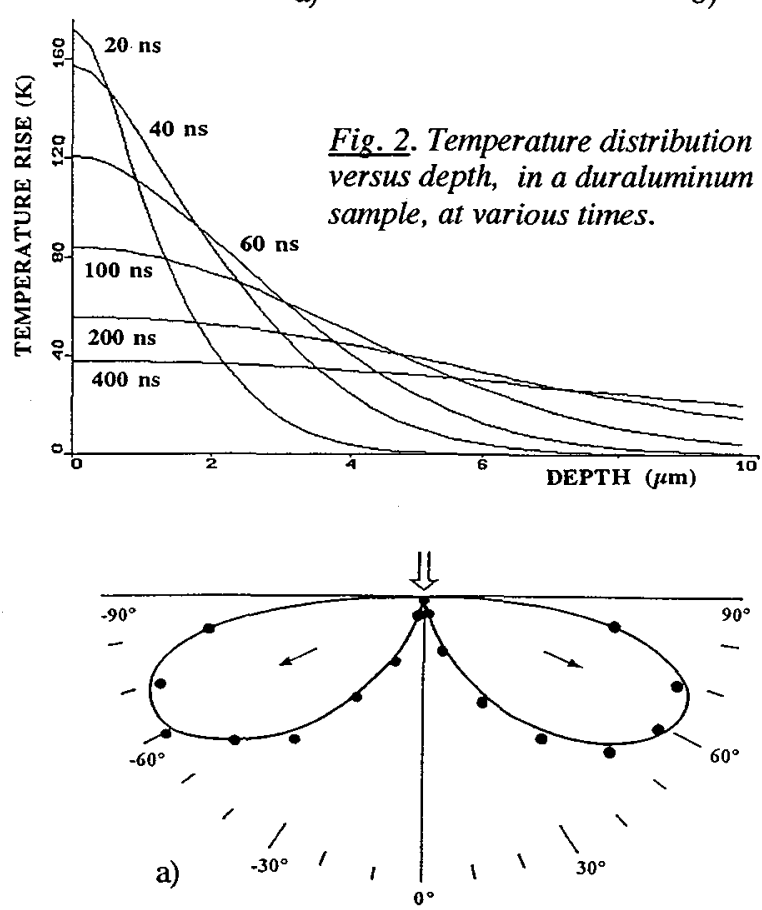

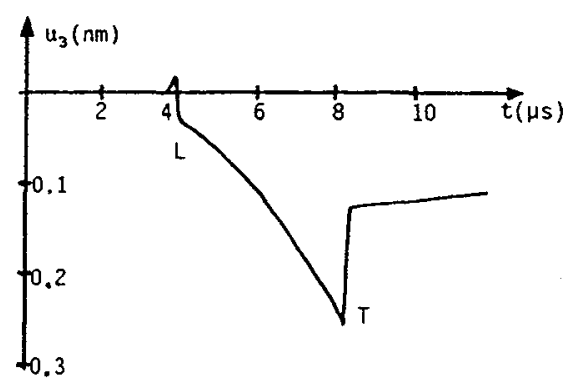

Fig. 3. Generation in a duraluminum plate of thickness $25 \mathrm{~mm}$, by a 40-mJ YAG laser pulse. Mechanical displacement computed at the epicentre.

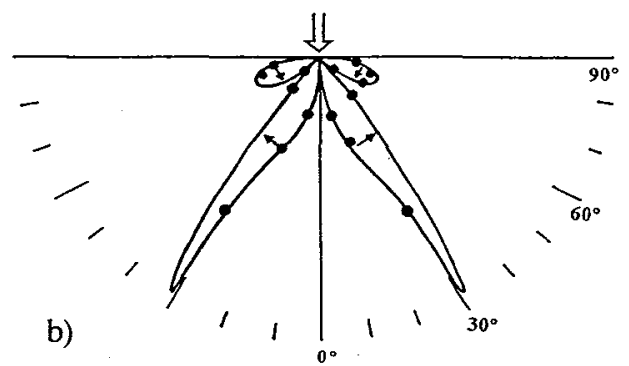

Fig. 4. Directivity pattern of the point thermoelastic source, a) longitudinal waves, b) shear waves, (•) experimental data [32].

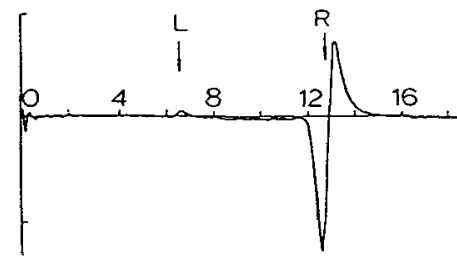

a)

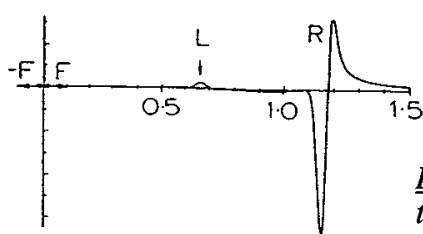

b)
Fig. 5. Rayleigh vaves $(R)$ generated in thermoelastic regime on the surface of a solid of Poisson coefficient $v=0.25$ : a) experiment, b) theory. 

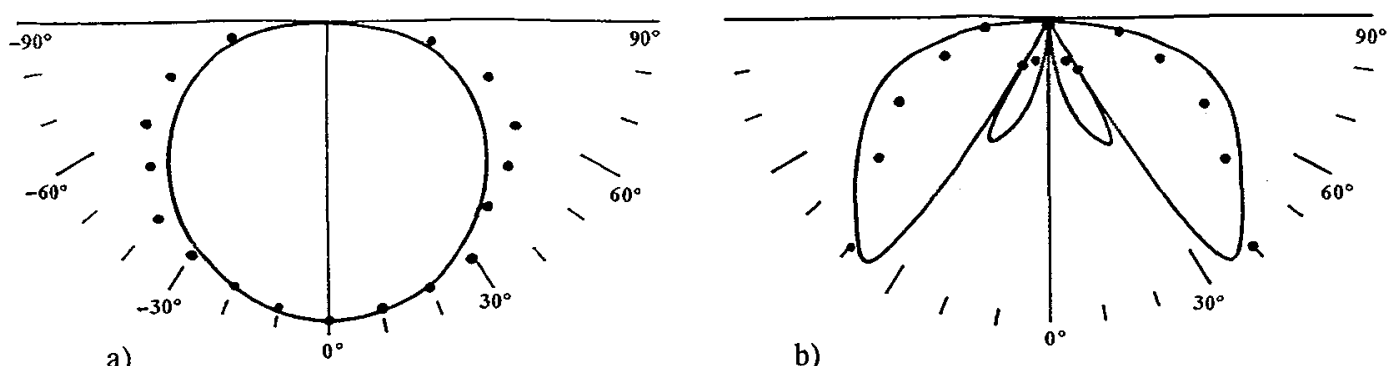

Fig. 6. Directivity pattern of a point source in the ablation regime. a) longitudinal waves, b) shear waves, $(\bullet)$ experimental data [32].
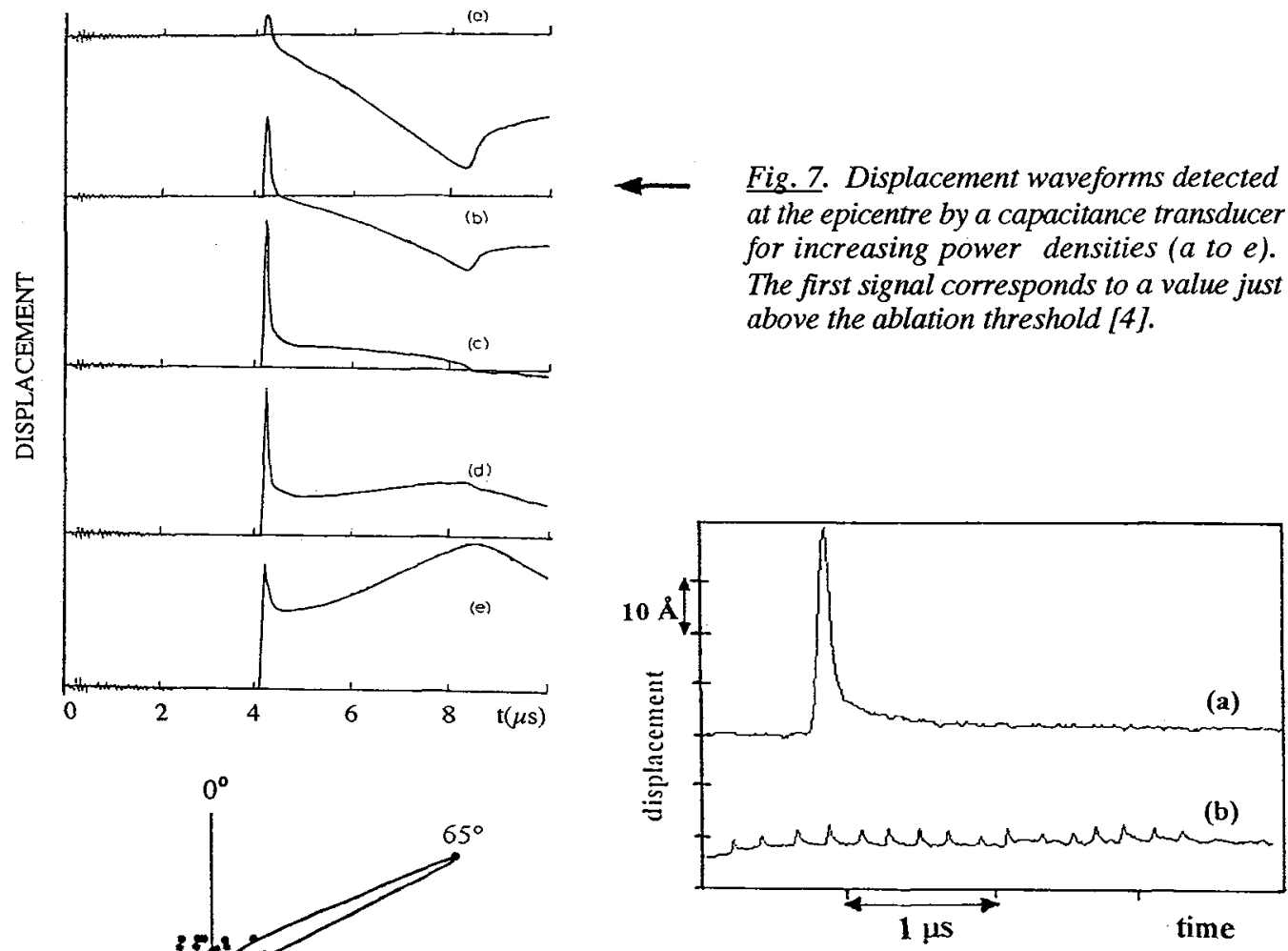

Fig. 8. Directivity pattern obtained with the 16 thermoelastic source array focussing power into direction $\theta=65^{\circ} .(\bullet)$ experimental data.

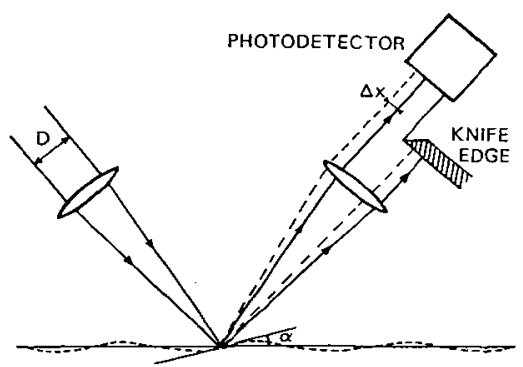

Fig. 7. Displacement waveforms detected at the epicentre by a capacitance transducer for increasing power densities (a to e). The first signal corresponds to a value just above the ablation threshold [4].

Fig. 9. Longitudinal acoustic displacements detected by an optical probe. a) at the focal point $(R=50 \mathrm{~mm}$, $\left.\theta=65^{\circ} . b\right)$ in the symmetrical direction $\left(\theta=-65^{\circ}\right)$ where no focusing exists.

Fig. 10. Knife edge technique. The ripple of the surface changes the direction of the reflected beam at the frequency of the surface acoustic wave. The wedge masking partially the oscillating beam, the photodetector output current is modulated. 


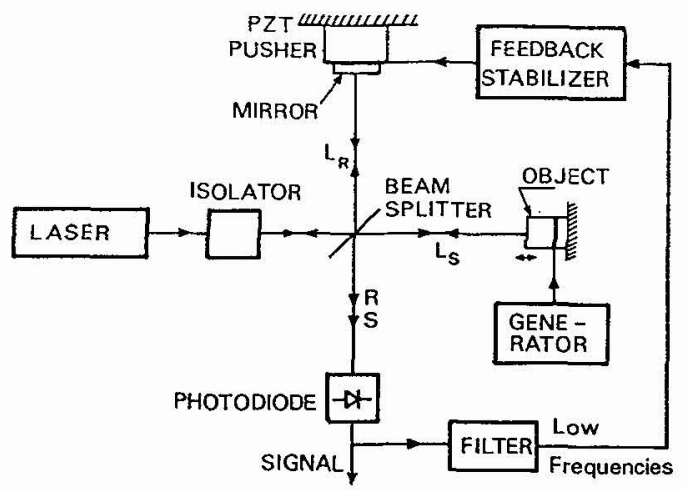

Fig. 11-Stabilized Michelson interferometer. The position of the reference mirror is controlled by the low frequency part of the photodiode signal in order to hold fixed the operating point in spite of the optical path fluctuations. The sensitivity is maximum for the optical path difference of beam $R$ and $S$ equal to $\mathrm{N} / 4$.
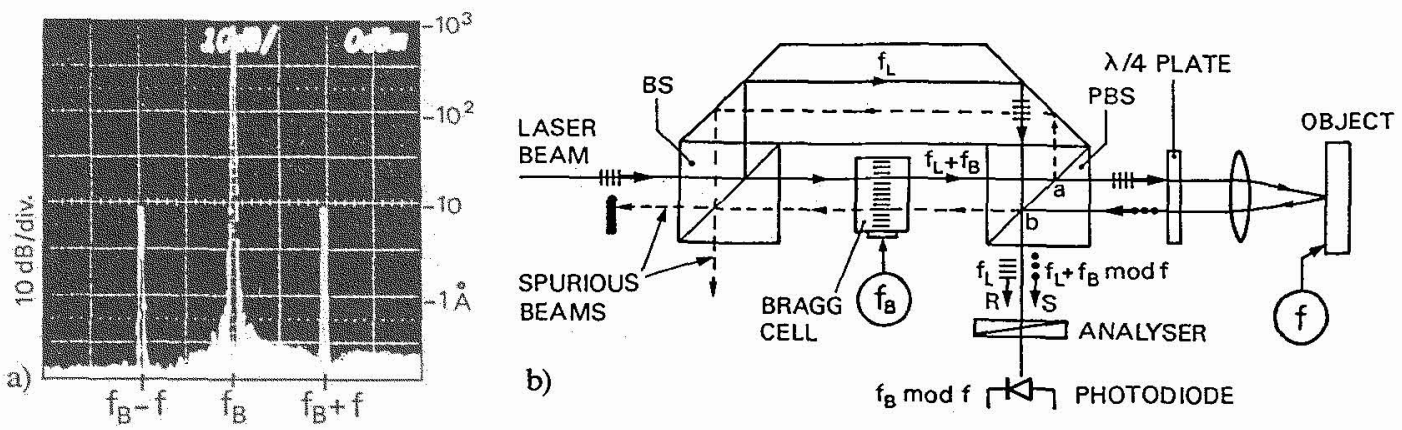

Fig. 12. Heterodyne interferometry. a) Spectrum of the photocurrent. b) Compact optical configuration.
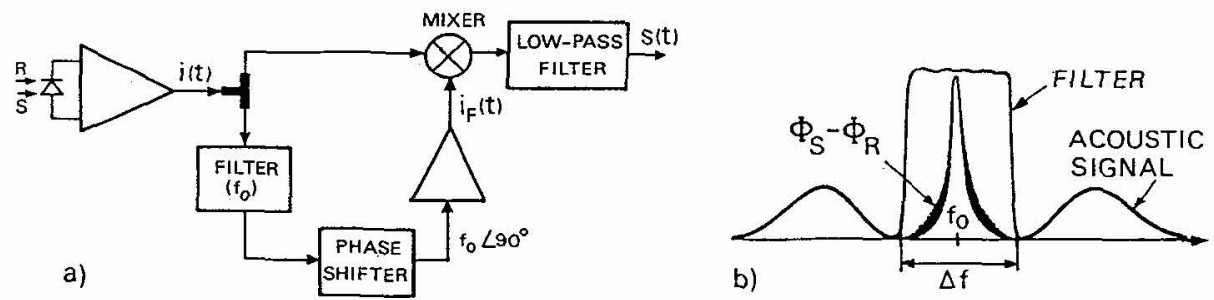

Fig. 13. Broadband coherent detection for an heterodyne probe. a) The mixing of the part of the photocurrent at carrier frequency fo, $\pi / 2$-phase shifted, with the other part, unchanged, provides the displacement. b) For the fluctuations $\Phi_{S}-\Phi_{R}$ to be cancelled, the bandpass of the filter is larger than their spectrum width.

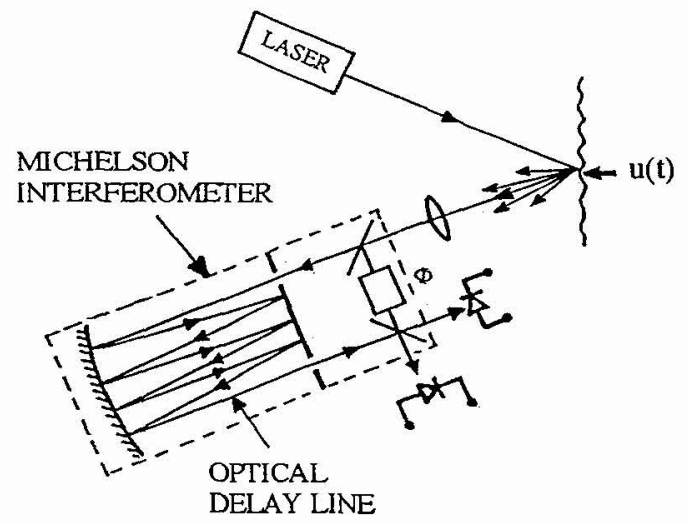

Fig. 14. Time-delay interferometry. The principle of this method consists of making the optical wave scattered by the surface beat with itself after a timedelay. 

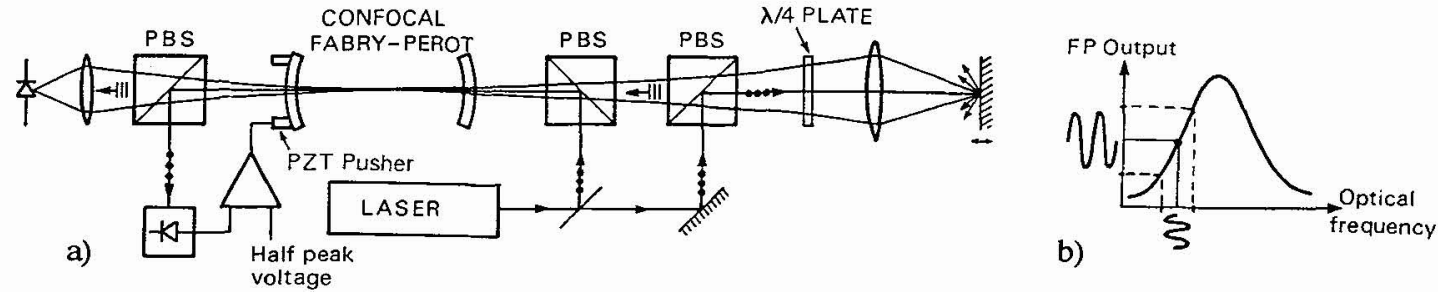

Fig. 15. Confocal Fabry-Pérot (FP) interferometer [24]. PBS is for polarizing beam splitter. The FP cavity is controlled by a piezoelectric pusher so that the laser frequency is maintained on the slope of one of the transmission peak.

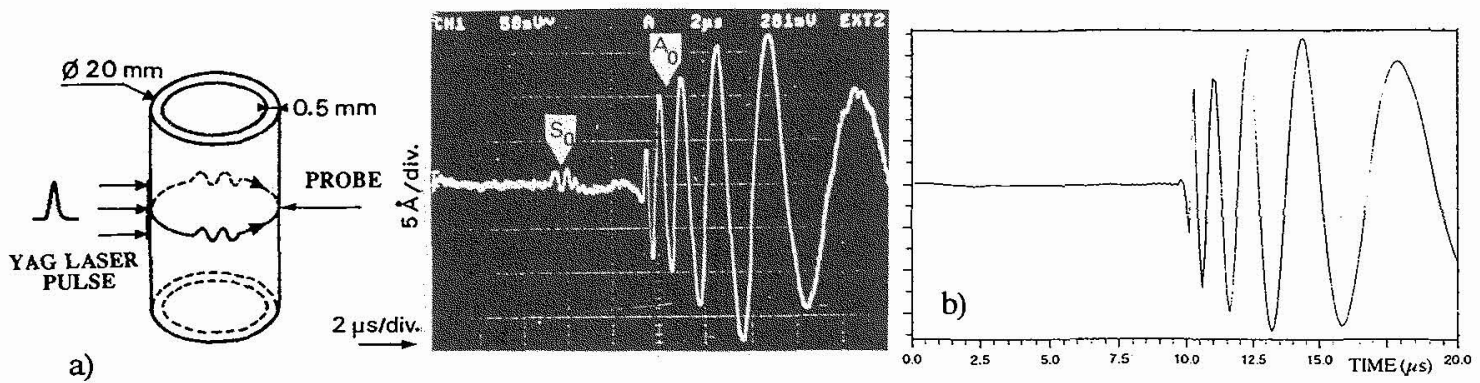

Fig. 16. Detection of Lamb waves generated in a hollow cylinder by a light pulse. The light source is a line. a) Experiment: the dispersive effect is easily observed. b) Computed waveform.
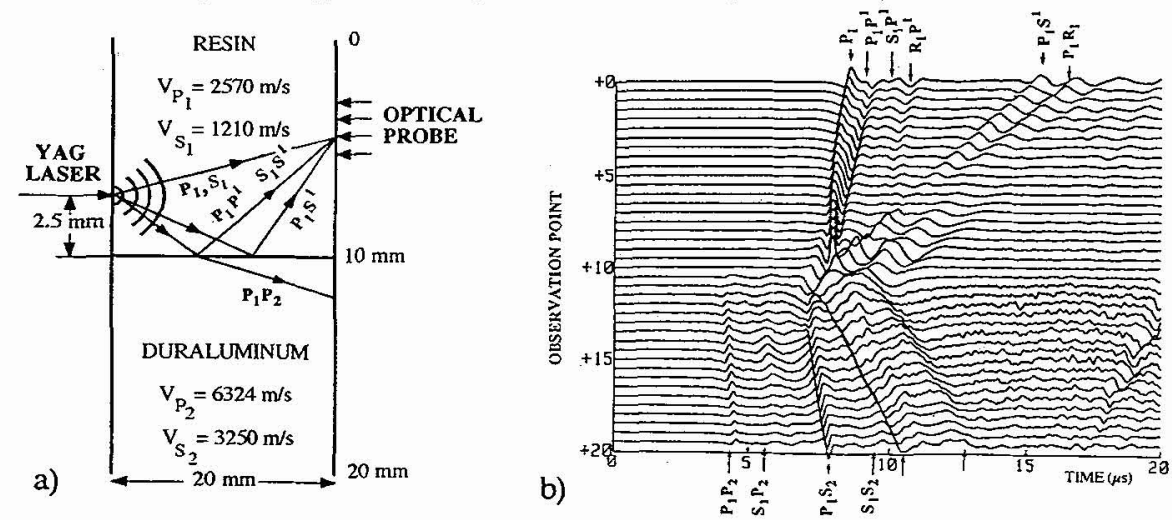

Fig. 17. Physical modeling of sismic prospecting experiments using laser ultrasonic techniques.
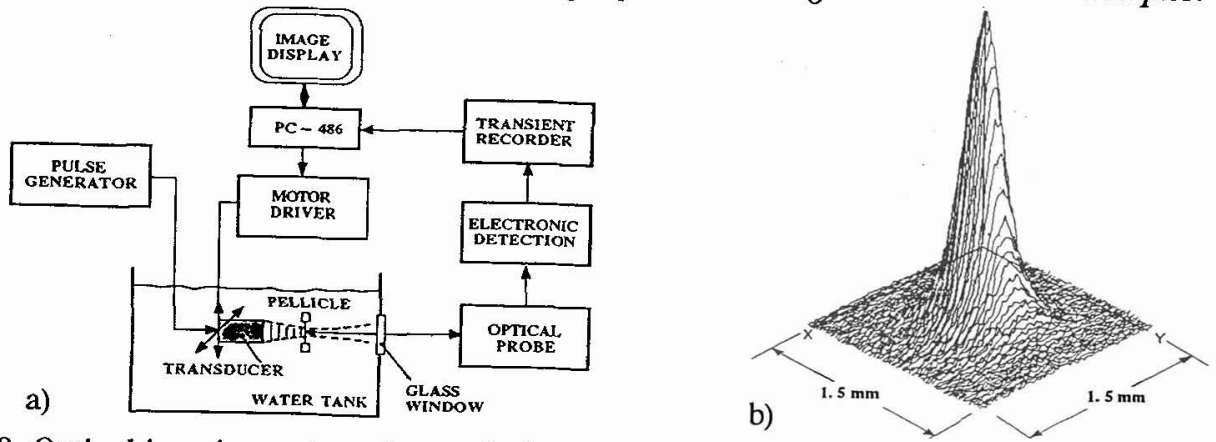

Fig. 18. Optical imaging system of acoustic fields in water. a) Experimental set-up. b) Acoustic field (displacement) transmitted by a $25-\mathrm{MHz}$ focused $(F=18 \mathrm{~mm})$ piezoelectric transducer, measured on a 6- $\mu$ m thick membrane of mylar. 